\title{
HYDRODYNAMIC MODELING OF MASS LOSS FROM CATACLYSMIC VARIABLE SECONDARIES
}

Ronald L. Gillijland**

Lick Observatory, Board of Studies in Astronomy and

Astrophysics, University of California, Santa Cruz, CA 95064

\section{ABSTRACT}

Results of detailed computer simulations of mass loss from a cataclysmic variable secondary are presented. The calculations involve solution of the nonlinear hydrodynamical equations of stellar structure under varying degrees of approximation in an attempt to determine stability of the mass loss process. For comparison with previous theoretical studies dynamical sequences were computed assuming spherically symmetric mass loss from the secondary. The assumption of spherical symmetry is very poor since nearly all of the transferred material is lost within a small region about the inner Lagrangian point. Dynamical sequences treating only the region near the Lagrangian point were constructed in a manner consistent with the assumption of Roche geometry. Finally the effects of mass flow nonorthogonal to the Roche equipotential surfaces were treated in a very simple way. The last generalization produces stable mass loss in a model which was unstable for the less realistic approximation schemes. The finding of stable mass transfer implies that instability of the secondary star is not the mechanism leading to cataclysmic variable outbursts. This conclusion is consistent with published observations of dwarf novae made just prior to outburst.

\footnotetext{
*A detailed account of this study will be published elsewhere.

**Now at National Center for Atmospheric Research, P. 0. Box 3000 , Boulder, CO 80307
} 


\section{DISCUSSION}

STARREIELD: I disagree with you regarding the hot spot. The observations do show that the hot spot gets more luminous during the outburst. A serious problem with the dwarf novae is the question of what bolometric corrections should be used.

GIILILAND: Warner says that in $\mathrm{H}-\mathrm{alpha}$, the hot spot does not increase. Bolometrically, we really can't say. It seems unlikely that you can hide all of the change in the bolometric correction term.

STARRFIELD: You see only a small part of the outburst in the observations.

GILLILAND: I agree with that. The simplest assumption is that if you see no change in the optical, which is the case, then there is little total change.

ROBINSON: I do not think that it is a straightforward picture. In reality, it is extremely complicated.

GILIILAND: The hot spot changes after the eruption, but immediately preceding and during the outburst, the hot spot doesn't change. If mass transfer to the secondary is causing it, then you need a precursor or a change right at the beginning.

STARRFIELD: You don't if you turn on slowly.

YOUNG: You were saying that a lot of these stars have accretion disks. We have indirect evidence which suggests very strongly that the flow rates are highly variable.

GILLILAND: I completely agree that the flow rates are going to be variable during the outburst.

YOUNG: We see them going up and down over a time scale of weeks with no outbursts.

HUGGINS: What are the mechanisms for the sharp rise that you get in your calculations?

GILLILAND: The mechanism is the release of recombination energy as material flows through the ionization zone. The binding energy of the material in the region below the inner Lagrangian point is very small. 